\title{
Cerebral disorders in the first 7 years of life in children born post-term: a cohort study
}

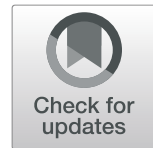

\author{
Anne Hald Rolschau ${ }^{1,2,3}$, Annette Wind Olesen ${ }^{4,5}$, Carsten Obel ${ }^{6}$, Jørn Olsen ${ }^{7}$, Chunsen S. Wu ${ }^{5,8}$ and \\ Poul-Erik Kofoed ${ }^{2,3^{*}}$ (iD
}

\begin{abstract}
Background: To estimate the association between post-term delivery and risk of physical disabilities, mental disabilities, and seizures during the first 7 years of life.

Methods: Data from 57,884 singleton infants born alive in week $39-45$ by mothers included in the Danish National Birth Cohort (1997 to 2004) were analyzed, of these 51,268 were born at term $(39-41+6)$ and 6616 post-term (42+ $0-44+6)$. Information on clinical endpoints was obtained from an interview at 18 months of gestational age, from a 7-year questionnaire, and from the Danish National Patient Register.

Logistic regression and Cox regression were used to estimate odds ratios and hazard rate ratios for the outcome obtained from the interview/questionnaire data and from the register-based data, respectively.

Results: We found no statistically significant increased risk of physical disabilities, mental disabilities, and epilepsy among children born post-term, though for most outcomes studied a tendency towards more adverse outcomes was seen. When children born late term (week 41) were compared to children born in week 42 or later the same tendency was found.
\end{abstract}

Conclusion: Post-term born children had a tendency to an excess risk of neurological disabilities as followed for up to 7 years of age.

Keywords: Children, Disability, Neurodevelopment, Developmental disability, Post-term delivery

Post-term delivery has been associated with increased perinatal mortality $[1,2]$ but newer studies do not support these findings, probably due to an improved management of post-term pregnancies [3, 4]. However, for unknown reasons post-term deliveries still seem to cause higher neonatal morbidity [2,3], but it has been hypothesized to be caused by a decreased placental function in late pregnancy.

An increased risk of non-optimal motor development by increasing gestational age after 41 weeks has been reported $[5,6]$ and adds to the results of Moster et al. finding a higher risk of cerebral palsy in children born at 42 weeks or later compared to children born at 40 weeks [7]. The cognitive abilities of children born post-term

\footnotetext{
* Correspondence: poul.erik.kofoed@rsyd.dk

${ }^{2}$ Institute of Regional Health Research, University of Southern Denmark, Odense, Denmark

${ }^{3}$ Department of Pediatrics, Lillebaelt Hospital, Kolding Hospital, Sygehusvej 24, 6000 Kolding, Denmark
}

Full list of author information is available at the end of the article have also been found to be negatively associated with a high gestational age at birth [8-10].

Post-term delivery has been shown to be a risk factor for seizures in the neonatal period [11] and during the first year of life [12]. Ehrenstein did, however not find evidence for an increased risk of epilepsy beyond the first year of life and until the end of the 12-years followup period [12], corroborating results from a Danish register-based study [4] that reported no increased risk for cerebral palsy, epilepsy, or sensorineural defects [4].

A cohort study showed that more 18 months old children born post-term achieved the assessed developmental milestones compared with children born at term (39-40 weeks) [13] and a Danish study showed a decreasing risk of impaired coordination development by increasing gestational age [14].

With respect to mental problems, a Dutch cohort study showed an increased risk of developing behavioral and emotional problems such as attention deficit 
hyperactivity disorder (ADHD) in children born postterm compared to children born at term when examined at 1.5 and 3 years of age [15] and an American study of children with autism spectrum disorders found a significantly higher Social Communication Questionnaire score in children born post-term ( $>42$ weeks) compared to children born at term, indicating a higher risk of autism spectrum disorders [16]. However, a meta-analysis of perinatal and neonatal risk factors for autism reported no association between autism and post-term birth [17].

The association between neurological complications and post-term deliveries is thus still uncertain. Therefore, further studies are needed in order to formulate evidence-based recommendations to those, who are responsible for the obstetric management of pregnancies exceeding into the post-term period. The aim of this study was to examine if post-term born children were at higher risk of physical disabilities, mental disabilities, or seizures at the age of 7 years compared to children born at term.

\section{Methods}

Data was retrieved from the Danish National Birth Cohort (DNBC), which is a follow-up study of about 100,000 children born in the period 1997 to 2004. The DNBC is estimated to have recruited about 30\% of the target population. About $50 \%$ of all general practitioners took part in the recruitment and $60 \%$ of those invited accepted the invitation [18]. The participants were interviewed by telephone twice before and twice after giving birth and asked to fill in a questionnaire when the child was 7 years old. The first and second interview at 12 and 30 weeks' gestation, respectively, included information on reproductive, toxicological, and socio-demographic factors, lifestyle factors, and health status before and during pregnancy. The third interview at 6 months after delivery included information on health status in the last part of the pregnancy and the development of the child. The fourth interview, 18 months after delivery, mainly added information on development and milestones of the child. In the questionnaire at 7 years of age information on developmental milestones, health status, physical skills, and eating habits were included (www.DNBC.dk).

Data from the interviews and the 7-year questionnaire were linked to data from the Danish National Patient Register.

Data included in this study was obtained from a cohort of 90,191 pregnancies where the mothers participated in the first pregnancy interview. Two women withdrew their informed consent to participate in the study. Subsequent pregnancies during the inclusion period, multiple gestations, stillbirth, abortion, and deliveries below gestational age $39+0$ weeks or above $45+0$ weeks were excluded. We identified 64,262 children born in gestational week $39-45(17,112$ born in week $39,23,313$ in week 40 , 16,531 in week 41, 6985 in week 42, 299 in week 43 and 22 in week 44). Of these, 2579 children born of mothers who reported chronic diseases [essential hypertension (O100-O109, O130-O139, I100-I109), preeclampsia (O140-O149), thyroid disease (O992O992a), diabetes type 1 (E100-E109), diabetes type 2 (E110-E119A), gestational diabetes (O240-O249)] according to the 10th revision codes of the International Statistical Classification of Diseases and Related Health Problems (ICD10) were excluded. Finally, 3799 cases of elective caesarean section (O820) or acute caesarean section before spontaneous labor (O821A) both at-term and post-term were excluded. Thus infants born after a caesarian section were only included if the caesarian was decided during delivery. This left 57,884 children for the final analysis (Fig. 1).

Data on gestational age was retrieved from the Danish National Patient Register. In the study period, the gestational age was estimated by ultrasound and used if the last menstrual period was considered unreliable [19]. Post-term delivery was defined as a pregnancy with delivery in gestational week $42+0$ to $44+6$, and term delivery as delivery in gestational week $39+0$ to $41+6$ (reference group).

\section{Outcome measures}

Outcomes of interest were extracted from the interview/ questionnaire obtained from the DNBC, and the Danish National Patient Register. Background and baseline information concerning the maternal health status and the pregnancy were obtained from the first interviews in the DNBC. The interview/questionnaire outcomes concerning the child were obtained from the DNBC including information on 1) physical disabilities (chronic disease or handicap from the 7-year questionnaire, and developmental deficit from the 18-months interview), 2) seizures (epilepsy and febrile seizures from the 7-year questionnaire), and 3) mental disabilities (speech development, late start at school, and psychiatric case status from the 7-year questionnaire).

For assessing psychiatric problems an extended version of the Strengths and Difficulties Questionnaire (SDQ) [20-22] was used to provide a measure of the overall status of behavioral or social difficulties. We used the predictive algorithme based on one informant to define psychiatric caseness. The algorithme is based on the 20 questions for the subscales: 1) emotional symptoms (5 items), 2) conduct problems (5 
Study population

Interview 1

90,191 pregnancies $(83,845$ women)

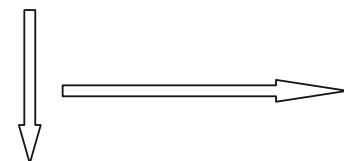

First pregnancies for each woman in the cohort

83,843

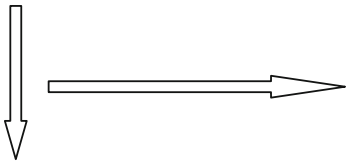

Live born singletons

80,659

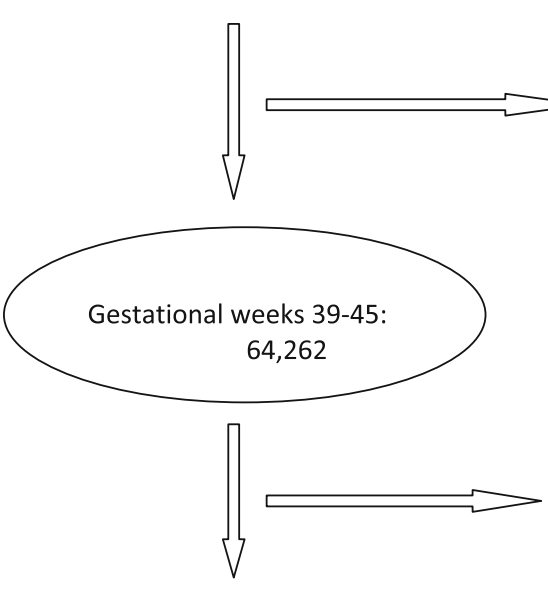

Final study population: 57,884

(51,268 term, 6,616 post-term)
Exclusion

Withdrew from study: 2

Subsequent pregnancies within

recruitment period: 6,346

Twin pregnancies: 1,811

Triplet pregnancies: 30

Stillbirth or abortion: 1,343

Gestational weeks $<39$ :

37-38: 12,049

$=<36: 3,669$

Gestational weeks >45: 51

Missing gestational age: 628
Mothers with chronic diseases: 2,579

Elective caesarean or acute caesarean before

onset of labour: 3,799

Fig. 1 Study population. Flow chart showing number of pregnancies and number of live born children included in the study

items), 3) hyperactivity/inattention (5 items), and 4) peer relationship problems (5 items), and the added score was combined with the score of daily impact. Based on this definition about $8 \%$ of the population was identified as having a possible psychiatric disorder.

Most of the answers to the remaining questions in the interview and the questionnaire were truncated as "yes"- or "no"-answers, i.e. "Has a doctor ever said that your child had epilepsy?"

Diagnoses based on the Danish version of the ICD10 were obtained from the Danish National Patient Register as recorded during the entire follow-up period. The diagnoses included physical disabilities as cerebral and other palsies (DG80.0-9, DG81.0-9, DG82.0-5, DG83.0-9), seizures as epilepsy (DG40.0-9, DG41.0-9) and febrile 
seizures (DR560, DR560A) and mental and psychiatric disabilities (DF40.0-9, DF42.0-9, DF80.0-DF83.9, DF90.0DF98.9), including infantile autism (F84.0-F89.9).

\section{Statistical analysis}

Differences in characteristics between term and postterm were compared using chi square test for categorical variables.

The children were followed from birth until the time of first recorded occurrence of the outcomes of interest (physical disabilities, mental disabilities, and seizures), emigration, death, or end of follow-up, whichever came first. The underlying time scale was age of the child in days. We used Cox proportional hazards models to estimate hazard rate ratios (HRs) with 95\% confidence intervals (95\% CI) for the onset of the outcomes registered in the Danish National Patient Register.

The analyses were conducted for each outcome, respectively. The results were adjusted for potential confounders including maternal age (continuous variable), parity $(0 / 1+)$, socio-occupational status (high, medium, and low), smoking (yes/no but partner smoking/no), pre-pregnancy body mass index (continuous variable), and child's sex (boy/girl).

The Cox proportional hazards assumptions were examined using log-log plots, which showed no severe violation of proportionality.

We conducted both crude as well as adjusted logistic regression to estimate odd ratios (ORs) with 95\% confidence intervals for data from DNBC.

Finally, in a sub-analysis, the outcome mesurements were estimated for children born post-term $(42+0$ $44+6,6616$ children) compared to late term $(41+0$ $41+6,15,390$ children) (as reference).

Statistical analyses were performed using STATA version 13 (Stata Corp., College Station, Texas, USA).

\section{Results}

Figure 1 illustrates how the 57,884 children participating in the final analysis were identified. In Table 1, maternal characteristics are shown. The group of women who delivered post-term more often gave birth to boys and was characterized by more nulliparas, and with higher prepregnancy body-mass-index. There were slightly fewer with fever during pregnancy. We found no significant differences in socio-occupational status, maternal age, or the frequency of maternal epilepsy, psychiatric disorder, folic acid consumption, smoking and alcohol consumption (Table 1). Very few admitted using hash (137/48, $345(0.3 \%)$ vs. $16 / 6279(0.3 \%))$ or other drugs during pregnancy $(20 / 48,354(0.04 \%)$ vs. $3 / 6281(0.05 \%))$.

No statistically significant differences were found between children born at term (gestational age $39+$
0 to $41+6)$ and the post-term born children $(42+0$ to $44+6)$ in risk of physical disabilities or seizures when analyzing the mother's answers at the 18month interview and the 7-year questionnaire. An increased risk of psychiatric caseness as evaluated by the SDQ questionnaire was seen in the postterm group (Table 2). When using data from the Danish National Patient Register no statistically significant differences were found for cerebral palsy, psychiatric diagnoses or epilepsy, whereas more in the post-term group got febrile seizures (Table 3). However, for most conditions, apart from epilepsy and psychiatric diagnoses when analyzed on data from the Danish National Patient Register, a tendency towards an increased risk was seen for the post-term group.

In a sub-analysis comparing the post-term group $(42+0-44+6)$ (as exposed) with the late term group $(41+0-41+6)$ (as reference), we found an increased risk of developmental deficit (OR 1,33; 95\%CI: 1.00-1.77), chronic disease or handicap (OR 1, 16; 95\%-CI: 1.00-1.35), and psychiatric caseness (OR 1.19; 95\%-CI: 1.04-1.36) in interview/questionnaire data from DNBC and a significantly increased risk of febrile seizures (HR 1.60; 95\%-CI: 1.09-2.33) using data from the Danish National Patient Register (Tables 4 and 5).

\section{Discussion}

Neurodevelopmental outcome of 51,268 infants born at term and 6616 born post-term were examined using data from DNBC and the Danish National Patient Register. No statistically significant increased risk of physical or mental disabilities was found in post-term born infants as compared to infants born at-term. For most of the conditions examined a tendency towards an increased risk was seen ( Tables 2 and 3).

A discrepancy in the results for febrile seizures was found as the mothers up to ten times more often stated that the child had experienced a febrile seizure than that reported in the Danish National Patient Register which may be due to underreporting in the national register $(0.5 \%, 272 / 56,711)$ as compared to self-reporting $(5.6 \%$, $2753 / 49,011)$, as the incidence of febrile seizures in Denmark is approximately 4\% [23]. Very few children had psychiatric diagnoses, whereas using the SDQ questionnaire $8.1 \%$ in the term group and $9.5 \%$ in the postterm group had a score indicating a psychiatric disorder, demonstrating that only the more severe cases had been diagnosed and thus reported to the Danish National Patient Register, whereas the SDQ is a screening for psychiatric and mental challenges covering more aspects of the psychiatric and mental wellbeing of the child. 
Table 1 Characteristics of 57,884 women who delivered at term $(39+0-41+6$ gestational weeks) or post-term $(42+0-44+6$ gestational weeks)

\begin{tabular}{llllll}
\hline & \multicolumn{2}{l}{ At term } & & \multicolumn{2}{l}{ Post-term } \\
& $\mathrm{n}$ & $\%$ & & $\%$ \\
\hline Total & 51,268 & 100.0 & & 6616 & 100.0
\end{tabular}

Maternal age

$\begin{array}{lllll}10-25 & 8846 & 17.3 & 1159 & 17.5 \\ 26-30 & 22,360 & 43.6 & 2862 & 43.3 \\ 31-35 & 15,696 & 30.6 & 2004 & 30.3 \\ 36-50 & 4366 & 8.5 & 591 & 8.9 \\ & & & & P=0.616\end{array}$

Parity

0

1

2

3

$4+$

Missing

Pre-pregnancy BMI

$<18.5$
$18.5-<25$
$25-<30$
$>30$
Missing

Child's sex

Male

Female

Missing

Socio-occupational status

$\begin{array}{lllll}\text { High } & 27,109 & 52.9 & 3511 & 53.1 \\ \text { Medium } & 19,206 & 37.5 & 2478 & 37.5 \\ \text { Low } & 4764 & 9.3 & 606 & 9.2 \\ \text { Missing } & 189 & 0.4 & 21 & 0.3 \\ & & & & P=0.902\end{array}$

Smoking

$\begin{array}{lllll}\text { Yes } & 7423 & 14.5 & 927 & 14.0 \\ \text { No, but partner smoking } & 7547 & 14.7 & 1057 & 16.0 \\ \text { No } & 24,839 & 48.4 & 3165 & 47.8 \\ \text { Missing } & 11,459 & 22.4 & 1467 & 22.2 \\ & & & & P=0.054 \\ & 39+0-41+6 & 42+0-44+6 \\ & n & \% & \text { N } & \%\end{array}$

Table 1 Characteristics of 57,884 women who delivered at term $(39+0-41+6$ gestational weeks) or post-term $(42+0-44+6$ gestational weeks) (Continued)

\begin{tabular}{|c|c|c|c|c|}
\hline & \multicolumn{2}{|c|}{ At term } & \multicolumn{2}{|c|}{ Post-term } \\
\hline & $\mathrm{n}$ & $\%$ & $\bar{N}$ & $\%$ \\
\hline \multicolumn{5}{|c|}{ Maternal epilepsy, medicated } \\
\hline Yes & 113 & 0.2 & 11 & 0.2 \\
\hline No & 39,723 & 77.5 & 5140 & 77.7 \\
\hline \multirow[t]{2}{*}{ Missing } & 11,432 & 22.3 & 1465 & 22.1 \\
\hline & & & & $P=0.638$ \\
\hline \multicolumn{5}{|c|}{ Psychiatric disorder } \\
\hline Yes & 3869 & 7.5 & 467 & 7.1 \\
\hline No & 47,357 & 92.4 & 6144 & 92.9 \\
\hline \multirow[t]{2}{*}{ Missing } & 42 & 0.1 & 5 & 0.1 \\
\hline & & & & $P=0.364$ \\
\hline \multicolumn{5}{|c|}{ Alcohol consumption during pregnancy } \\
\hline Yes & 9351 & 18.2 & 1199 & 18.1 \\
\hline No & 30,393 & 59.3 & 3939 & 59.5 \\
\hline \multirow[t]{2}{*}{ Missing } & 11,524 & 22.5 & 1478 & 22.3 \\
\hline & & & & $P=0.924$ \\
\hline
\end{tabular}

Fever during pregnancy

$\begin{array}{lllll}\text { Yes } & 11,138 & 21.7 & 1390 & 21.0 \\ \text { No } & 36,898 & 72.0 & 4848 & 73.3 \\ \text { Missing } & 3232 & 6.3 & 378 & 5.7 \\ & & & & P=0.049\end{array}$

Folic acid consumption during pregnancy

\begin{tabular}{lllll} 
Yes & 12,070 & 23.5 & 1567 & 23.7 \\
No & 36,223 & 70.7 & 4707 & 71.1 \\
Missing & 175 & 0.3 & 12 & 0.2 \\
& & & & $P=0.062$ \\
\hline
\end{tabular}

We compared the outcome of post-term children with that of children born at late term and found a higher risk of adverse outcomes supporting the present recommendation in Denmark for induction of births at gestational age $41+3$, and thus giving birth before week $42+0$.

Most women who delivered post-term were healthy and had pregnancies without complications. The effect on the infant of being born post-term could at least in part be due to the increased exposure to the intrauterine environment. Indication for induction in this cohort was a prolonged pregnancy reducing the risk of selection bias and confounding by indication.

The major limitation of the study is in the nature of observational research where unknown confounders may have masked or exaggerated differences, why there is a 
Table 2 Physical disabilities, mental disabilities, and seizures in children born post-term compared to children born at term. Data from the 7-year interview (DNBC)

\begin{tabular}{|c|c|c|c|c|}
\hline Variable & $\begin{array}{l}\text { Logistic regression } \\
\text { OR }\end{array}$ & $\begin{array}{l}\text { Adjusted logistic regression } \\
\text { OR }(95 \% \mathrm{Cl})\end{array}$ & $\mathrm{n}_{1} / \mathrm{N}_{1}^{\mathrm{b}}$ & $\mathrm{n}_{2} / \mathrm{N}_{2}^{\mathrm{c}}$ \\
\hline Developmental deficit $^{d}$ & 1.09 & $1.12(0.88-1.43)$ & $542 / 50,239$ & $76 / 6465$ \\
\hline Chronic disease or handicap & 1.14 & $1.10(0.97-1.25)$ & $1937 / 50,027$ & $278 / 6424$ \\
\hline Speech development & 1.12 & $1.11(0.99-1.25)$ & $2667 / 30,619$ & $374 / 3898$ \\
\hline Late start at school & 1.03 & $0.99(0.74-1.33)$ & $383 / 30,687$ & $52 / 3905$ \\
\hline Case-caseness SDQ & 1.18 & $1.14(1.02-1.28)$ & $2494 / 30,750$ & $372 / 3914$ \\
\hline Epilepsy & 1.09 & $1.14(0.72-1.80)$ & $146 / 29,994$ & $21 / 3826$ \\
\hline Medication for epilepsy & 1.36 & $1.38(0.80-2.40)$ & $105 / 2041$ & $16 / 228$ \\
\hline Febrile seizures & 1.07 & $1.02(0.90-1.15)$ & $2430 / 43,437$ & $323 / 5574$ \\
\hline
\end{tabular}

OR Odds ratio calculated by logistic regression analysis

a adjusted for maternal age, parity, pre-pregnancy BMI, child's sex, and socio-occupational status

${ }^{b}$ number $\left(n_{1}\right)$ of cases amongst children born at term $\left(N_{1}\right)$ in adjusted logistic regression analysis

c number $\left(\mathrm{n}_{2}\right)$ of cases amongst children born post-term $\left(\mathrm{N}_{2}\right)$ in adjusted logistic regression analysis

d from 18 months interview

possibility of residual confounding. Misclassification of gestational age could potentially bias the results in both directions, but it is less likely in this data set with good quality gestational age data.

We had a large study population with close follow up. However, the low incidence of some of the studied outcomes limited our power. On the other hand, the large study-population also revealed even small to moderate differences with limited clinical relevance.

We excluded mothers with chronic diseases as their deliveries were often induced at term and could confound our results. Acute caesareans instituted before the onset of spontaneous labor are mostly due to maternal factors and were therefore censured as were all elective caesareans in order to compare the outcome of infants born spontaneously at term with infants born after pregnancies allowed to progress post-term. The results were adjusted for confounding by known risk factors of postterm delivery $[24,25]$.
However, ideally an unbiased study on the health consequences for the child of being born post term would require a randomized controlled trial set up. Pregnant women reaching term could be randomized to active or passive follow up. As such a study would hardly be acceptable we study interventions done by clinicians which make us susceptible to 'confounding by indication'. Those who have their pregnancy induced may have more risk factors than those who are allowed to proceed to become post-term.

The DNBC has previously been shown not to have significant selection bias for severe outcomes [26]. The combination with the Danish National Patient Register furthermore reduced the risk of bias due to differential loss to follow up, which can be a problem in a prospective cohort that does not include an entire population [27].

Information on gestational age was obtained from the Medical Birth Register, and usually based on ultrasound examination. The precision of gestational

Table 3 Physical disabilities, mental disabilities, and seizures in children born post-term compared to children born at term. Data from the Danish National Patient Register

\begin{tabular}{|c|c|c|c|c|}
\hline Variable & $\begin{array}{l}\text { Cox regression } \\
\text { HR }\end{array}$ & $\begin{array}{l}\text { Adjusted cox regression }{ }^{\text {a }} \\
\text { HR (95\% Cl) }\end{array}$ & $\mathrm{n}_{1} / \mathrm{N}_{1}^{\mathrm{b}}$ & $\mathrm{n}_{2} / \mathrm{N}_{2}^{\mathrm{c}}$ \\
\hline Cerebral and other palsies & 1.15 & $1.09(0.63-1.87)$ & $106 / 50,021$ & $15 / 6437$ \\
\hline Psychiatric diagnoses & 0.52 & $0,51(0.26-1.00)$ & $128 / 50,021$ & 9/6437 \\
\hline Autism & - & - & $4 / 51,268$ & $0 / 6616$ \\
\hline Epilepsy & 0.28 & $0.30(0.04-2.20)$ & $28 / 47,970$ & $1 / 6248$ \\
\hline Febrile seizures & 1.56 & $1.54(1.12-2.12)$ & $226 / 50,245$ & $46 / 6466$ \\
\hline
\end{tabular}

$H R$ Hazard ratio calculated by cox regression analysis

a adjusted for maternal age, parity, pre-pregnancy BMI, child's sex and socio-occupational status

${ }^{b}$ number $\left(n_{1}\right)$ of cases amongst children born at term $\left(N_{1}\right)$

${ }^{c}$ number $\left(\mathrm{n}_{2}\right)$ of cases amongst children born post-term $\left(\mathrm{N}_{2}\right)$ 
Table 4 Physical disabilities, mental disabilities, and seizures in children born post-term in week 42 or later compared to that of children born in week 41. Data from the 7-year interview (DNBC)

\begin{tabular}{|c|c|c|c|c|}
\hline Variable & $\begin{array}{l}\text { Logistic regression } \\
\text { OR }\end{array}$ & $\begin{array}{l}\text { Adjusted logistic regression }{ }^{a} \\
\text { OR }(95 \% \mathrm{Cl})\end{array}$ & $\mathrm{n}_{1} / \mathrm{N}_{1}^{\mathrm{b}}$ & $\mathrm{n}_{2} / \mathrm{N}_{2}^{\mathrm{c}}$ \\
\hline Developmental deficit $^{d}$ & 1.31 & $1.33(1.00-1.77)$ & $138 / 15,086$ & $76 / 6465$ \\
\hline Chronic disease or handicap & 1.21 & $1.16(1.00-1.35)$ & $551 / 15,010$ & $278 / 6424$ \\
\hline Speech development & 1.14 & $1.12(0.98-1.28)$ & 794/9169 & $374 / 3898$ \\
\hline Late start at school & 1.06 & $1.00(0.72-1.40)$ & 113/9149 & $52 / 3890$ \\
\hline Psychiatric caseness SDQ & 1.23 & $1.19(1.04-1.36)$ & $725 / 9204$ & $372 / 3914$ \\
\hline Epilepsy & 1.09 & $1.17(0.69-1.99)$ & $43 / 8934$ & $21 / 3811$ \\
\hline Medication for epilepsy & 1.51 & $1.51(0.80-2.87)$ & 29/610 & $16 / 228$ \\
\hline Febrile seizures & 1.05 & $1.00(0.87-1.14)$ & $739 / 13,023$ & $323 / 5574$ \\
\hline
\end{tabular}

OR Odds ratio calculated by logistic regression analysis

a adjusted for maternal age, parity, pre-pregnancy BMl, child's sex and socio-occupational status

${ }^{b}$ number $\left(n_{1}\right)$ of cases amongst children born $41+0-41+6\left(N_{1}\right)$ in adjusted logistic regression analysis

c number $\left(\mathrm{n}_{2}\right)$ of cases amongst children born $42+0-44+6\left(\mathrm{~N}_{2}\right)$ in adjusted logistic regression analysis

d from 18 months interview

age after introduction of routine ultrasound scans in Denmark is high, and even the last menstrual period based gestational age is often valid [25]. Routine ultrasound scans were used from the early 1990'ies in case of unreliable last menstrual period, and the frequency of routine ultrasound screening for malformations increased from 40 to $54 \%$ from 1989 to 90 to 1994-95, both increasing the validity of the data on gestational age at birth [19].

\section{Interpretation}

The analysis of several different variables increased the risk of finding significant associations due to chance and replication studies are thus needed. We found neither statistically significant adverse nor protective long-term effects on the studied outcomes when comparing post-term born children with all children born at term or with children born in week 41. However, both compared to all children born at term and children born in week 41 a non-significant tendency towards an adverse effect was seen for most studied outcomes. This corroborates the findings of a large study on the association between school performance at the age of 16 years and gestational age reporting a decreased performance for children born post-term after week 41 [28], and of a study finding increased intellectual disabilities with increasing gestational age post-term from week 42, an association persisting in a cohort of matched siblings suggesting that they were robust against confounding by shared familial traits [29].

\section{Conclusion}

Post-term delivery is considered an independent risk factor for neonatal morbidity [3], but long-term neurodevelopmental consequences have been less well studied. In this large observational study, a tendency to an increased risk of the studied outcomes in post-term children was found following the obstetric practice in Denmark at that time.

Table 5 Physical disabilities, mental disabilities, and seizures in children born post-term in week 42 or later compared to that of children born in week 41. From the Danish National Patient Register

\begin{tabular}{|c|c|c|c|c|}
\hline Variable & $\begin{array}{l}\text { Cox regression } \\
\text { HR }\end{array}$ & $\begin{array}{l}\text { Adjusted cox regression } \\
\text { HR }(95 \% \mathrm{Cl})\end{array}$ & $\mathrm{n}_{1} / \mathrm{N}_{1}^{\mathrm{b}}$ & $\mathrm{n}_{2} / \mathrm{N}_{2}^{\mathrm{c}}$ \\
\hline Cerebral and other palsies & 1.77 & $1.66(0.85-3.22)$ & $21 / 14,428$ & $15 / 6219$ \\
\hline Psychiatric diagnoses & 0.58 & $0,60(0.29-1.25)$ & $34 / 15,023$ & $9 / 6437$ \\
\hline Autism & & & $1 / 48$ & $0 / 0$ \\
\hline Epilepsy & 0.29 & $0.29(0.04-2.29)$ & $8 / 12,649$ & $1 / 5495$ \\
\hline Febrile seizures & 1.59 & $1.60(1.09-2.33)$ & $66 / 15,086$ & $46 / 6466$ \\
\hline
\end{tabular}

HR Hazard ratio calculated by cox regression analysis

a adjusted for maternal age, parity, pre-pregnancy BMI, child's sex and socio-occupational status

${ }^{b}$ number $\left(n_{1}\right)$ of cases amongst children born $41+0-41+6\left(N_{1}\right)$

c number $\left(\mathrm{n}_{2}\right)$ of cases amongst children born $42+0-44+6\left(\mathrm{~N}_{2}\right)$ 


\section{Abbreviations}

ADHD: Attention deficit hyperactivity disorder; DNBC: Danish National Birth Cohort; ICD10: The International Statistical Classification of Diseases and Related Health Problems 10th Revision; SDQ: Strengths and Difficulties Questionnaire

\section{Acknowledgements}

We thank the Danish National Board of Health for access to data linkage to the Medical Birth Registry and the Danish National Discharge Register.

\section{Authors' contributions}

AHR, AWO, JO and PK: designed the study. AHR, AWO and CSW: did the analyses. AHR, AWO, JO, CO and PK: participated in the interpretation of data. AHR and PK drafted the paper. AHR, AWO, JO, CO, CSW and PK revised it critically, and all approved the final version of the paper.

\section{Funding}

The study was supported by Ole Kirk's Fond, the Department of Pediatrics, and the Department of Gynecology and Obstetrics, Lillebaelt Hospital, Kolding. None of the funders had any role in the design of the study and collection, analysis, and interpretation of data or in writing the manuscript.

\section{Availability of data and materials}

The data that support the findings of this study are available from Bedre Sundhed i Generationer (BSIG.dk) but restrictions apply to the availability of these data, which were used under license for the current study, and so are not publicly available. Data are however available from the authors upon reasonable request and with permission of Bedre Sundhed i Generationer (BSIG.dk).

\section{Ethics approval and consent to participate}

The study is approved by the Danish Data Protection Agency. The data collection in the Danish National Birth Cohort is approved by the Regional Scientific Ethics Committee for the Municipalities of Copenhagen and Frederiksberg (KF 01-471/94). The participant gave written consent to being included in the Danish National Birth Cohort (www.DNBC.dk) and their data can any time they want be deleted from the study (https://www.bsig.dk/tildeltagerne/udmeldelse).

\section{Consent for publication}

Not applicable.

\section{Competing interests}

The authors declare that they have no competing interests.

\section{Author details}

'Department of Gynecology and Obstetrics, Lillebaelt Hospital, Kolding, Denmark. ${ }^{2}$ Institute of Regional Health Research, University of Southern Denmark, Odense, Denmark. ${ }^{3}$ Department of Pediatrics, Lillebaelt Hospital, Kolding Hospital, Sygehusvej 24, 6000 Kolding, Denmark. ${ }^{4}$ Department of Gynecology and Obstetrics, Odense University Hospital, Odense, Denmark. ${ }^{5}$ Institute of Clinical Research, University of Southern Denmark, Odense, Denmark. ${ }^{6}$ Research Unit for Mental Public Health, Institute of Public Health, Aarhus University, Aarhus, Denmark. ${ }^{7}$ Department of Clinical Epidemiology, Aarhus University, Aarhus, Denmark. ${ }^{8}$ Research Unit on Gynecology and Obstetrics, Odense University Hospital, Odense, Denmark.

Received: 16 July 2019 Accepted: 29 January 2020

Published online: 03 February 2020

\section{References}

1. Shea KM, Wilcox AJ, Little RE. Postterm delivery: a challenge for epidemiologic research. Epidemiology (Cambridge, Mass). 1998;9(2):199-204.

2. Olesen AW, Westergaard JG, Olsen J. Perinatal and maternal complications related to postterm delivery: a national register-based study, 1978-1993. Am J Obstet Gynecol. 2003;189(1):222-7.

3. Linder $\mathrm{N}$, Hiersch L, Fridman $\mathrm{E}$, et al. Post-term pregnancy is an independent risk factor for neonatal morbidity even in low-risk singleton pregnancies. Arch Dis Child Fetal Neonatal Ed. 2017;102(4):F286-f90.

4. Seikku L, Gissler M, Andersson S, Rahkonen P, Stefanovic V, Tikkanen M, Paavonen J, Rahkonen L. Asphyxia, Neurologic Morbidity, and Perinatal Mortality in Early-Term and Postterm Birth. Pediatrics. 2016;137(6). https:// doi.org/10.1542/peds.2015-3334. PubMed PMID: 27235446.
5. Vayssiere C, Haumonte JB, Chantry A, et al. Prolonged and post-term pregnancies: guidelines for clinical practice from the French College of Gynecologists and Obstetricians (CNGOF). Eur J Obstet Gynecol Reprod Biol. 2013;169(1):10-6.

6. van Batenburg-Eddes $T$, de Groot $L$, Arends $L$, et al. Does gestational duration within the normal range predict infant neuromotor development? Early Hum Dev. 2008;84(10):659-65.

7. Moster D, Wilcox AJ, Vollset SE, Markestad T, Lie RT. Cerebral palsy among term and postterm births. Jama. 2010;304(9):976-82.

8. Yang S, Platt RW, Kramer MS. Variation in child cognitive ability by week of gestation among healthy term births. Am J Epidemiol. 2010;171(4):399-406.

9. MacKay DF, Smith GC, Dobbie R, Pell JP. Gestational age at delivery and special educational need: retrospective cohort study of 407,503 schoolchildren. PLoS Med. 2010;7(6):e1000289.

10. Lindstrom K, Fernell E, Westgren M. Developmental data in preschool children born after prolonged pregnancy. Acta paediatrica (Oslo, Norway : 1992). 2005;94(9):1192-7.

11. Glass HC, Pham TN, Danielsen B, Towner D, Glidden D, Wu YW. Antenatal and intrapartum risk factors for seizures in term newborns: a populationbased study, California 1998-2002. J Pediatr. 2009;154(1):24-8.e1.

12. Ehrenstein V, Pedersen L, Holsteen V, Larsen H, Rothman KJ, Sorensen HT. Postterm delivery and risk for epilepsy in childhood. Pediatrics. 2007;119(3):e554-61.

13. Olesen AW, Olsen J, Zhu JL. Developmental milestones in children born post-term in the Danish National Birth Cohort: a main research article. BJOG. 2015;122(10):1331-9.

14. Zhu JL, Olsen J, Olesen AW. Risk for developmental coordination disorder correlates with gestational age at birth. Paediatr Perinat Epidemiol. 2012; 26(6):572-7

15. El Marroun $\mathrm{H}$, Zeegers M, Steegers EA, et al. Post-term birth and the risk of behavioural and emotional problems in early childhood. Int J Epidemiol. 2012;41(3):773-81.

16. Movsas $\mathrm{TZ}$, Paneth $\mathrm{N}$. The effect of gestational age on symptom severity in children with autism spectrum disorder. J Autism Dev Disord. 2012;42(11):2431-9.

17. Gardener H, Spiegelman D, Buka SL. Perinatal and neonatal risk factors for autism: a comprehensive meta-analysis. Pediatrics. 2011;128(2):344-55.

18. Olsen J, Melbye M, Olsen SF, et al. The Danish National Birth Cohort--its background, structure and aim. Scand J Public Health. 2001;29(4):300-7.

19. Jorgensen FS. Epidemiological studies of obstetric ultrasound examinations in Denmark 1989-1990 versus 1994-1995. Acta Obstet Gynecol Scand. 1999; 78(4):305-9.

20. Goodman R. The extended version of the strengths and difficulties questionnaire as a guide to child psychiatric caseness and consequent burden. J Child Psychol Psychiatry, Allied Discip. 1999;40(5):791-9.

21. Goodman A, Lamping DL, Ploubidis GB. When to use broader internalising and externalising subscales instead of the hypothesised five subscales on the strengths and difficulties questionnaire (SDQ): data from British parents, teachers and children. J Abnorm Child Psychol. 2010;38(8):1179-91.

22. Schoemaker MM, Flapper B, Verheij NP, Wilson BN, Reinders-Messelink HA, de Kloet A. Evaluation of the developmental coordination disorder questionnaire as a screening instrument. Dev Med Child Neurol. 2006;48(8):668-73.

23. Vestergaard $\mathrm{M}$, Christensen J. Register-based studies on febrile seizures in Denmark. Brain and Development. 2009;31:372-7.

24. Roos N, Sahlin L, Ekman-Ordeberg G, Kieler H, Stephansson O. Maternal risk factors for postterm pregnancy and cesarean delivery following labor induction. Acta Obstet Gynecol Scand. 2010;89(8):1003-10.

25. Olesen AW, Westergaard JG, Olsen J. Prenatal risk indicators of a prolonged pregnancy. The Danish birth cohort 1998-2001. Acta Obstet Gynecol Scand. 2006;85(11):1338-41.

26. Nohr EA, Frydenberg M, Henriksen TB, Olsen J. Does low participation in cohort studies induce bias? Epidemiology (Cambridge, Mass). 2006;17(4):413-8.

27. Greene N, Greenland S, Olsen J, Nohr EA. Estimating bias from loss to follow-up in the Danish National Birth Cohort. Epidemiology (Cambridge, Mass). 2011;22(6):815-22.

28. Abel $\mathrm{K}$, Heuvelman $\mathrm{H}$, Wicks $\mathrm{S}$, et al. Gestational age at birth and academic performance: population-based cohort study. Int J Epidemiol. 2017;46(1):324-35.

29. Heuvelman H, Abel K, Wicks S, Gardner R, Johnstone E, Lee B, Magnusson C, Dalman C, Rai D. Gestational age at birth and risk of intellectual disability without a common genetic cause. Eur J Epidemiol. 2018;33(7):667-78.

\section{Publisher's Note}

Springer Nature remains neutral with regard to jurisdictional claims in published maps and institutional affiliations. 